\title{
Binding of MMP-9-degraded fibronectin to $\beta 6$ integrin promotes invasion via the FAK-Src-related Erk1/2 and PI3K/Akt/Smad-1/5/8 pathways in breast cancer
}

\author{
WENTONG LI ${ }^{1 *}$, ZHIJUN LIU $^{2 *}$, CHUNLING ZHAO ${ }^{2}$ and LIMIN ZHAI ${ }^{1}$ \\ Departments of ${ }^{1}$ Pathology and ${ }^{2}$ Medical Biology, Weifang Medical University, \\ Weifang, Shandong 261053, P.R. China
}

Received March 27, 2015; Accepted June 16, 2015

DOI: $10.3892 /$ or.2015.4103

\begin{abstract}
Fibronectin (FN) has been recognized as the key element in promoting cell adhesion, migration and oncogenic transformation. $\alpha v \beta 6$ integrin binds with $F N$ in an RGD-dependent manner and is associated with invasion and poor prognosis in many types of cancers. The extracellular matrix (ECM) is commonly degraded and becomes disorganized in cancers. Previous studies have shown that FN can be degraded into fragments by MMP-9 in vitro; MMP-9 expression is upregulated in breast cancer, therefore, the role of degraded FN in breast cancer progression needs to be investigated. In the present study, expression of $\beta 6$ integrin in breast cancer tissues was analyzed. The data were quite consistent with the hypothesis that $\beta 6$ integrin expression is an indicator of poor prognosis. Cell surface expression of $\beta 6$ integrin was correlated with the invasive behavior of the breast cancer cell lines. MMP-9-degraded FN was used to explore its effects on cell invasion and expression of $\beta 6$ integrin. The results indicated that MMP-9-degraded FN can adjust constituents of the $\alpha v \beta 6$ heterodimers at the early phase and significantly elevate amounts of $\beta 6$ integrin subunits at a later period. To better elucidate the mechanism by which $\beta 6$ integrin regulates FN-induced cell migration and invasion, we determined the expression and activity of the downstream kinases of $\beta 6$ integrin, and elucidated that focal adhesion kinase (FAK)-Src interaction promoted the invasion and migration of breast cancer cells after treatment of MMP-9-degraded FN through the Erk1/2 and PI3K/Akt/Smad-1/5/8 pathways.
\end{abstract}

\section{Introduction}

Numerous studies have shown that integrin expression profiles are subject to change during cancer growth and progression,

Correspondence to: Dr Wentong Li, Department of Pathology, Weifang Medical University, Weifang, Shandong 261053, P.R. China E-mail: liwentong11@163.com

${ }^{*}$ Contributed equally

Key words: $\beta 6$ integrin, fibronectin, MMP-9, invasion, breast cancer and these changes contribute to the aggressive behavior of cancer cells $(1,2)$. Notably, integrins also play important roles in the tumor microenvironment and significantly contribute to tumor progression (3). The $\alpha v \beta 6$ integrin is an epithelial restricted transmembrane protein and is upregulated in many tumor types, including pancreatic ductal cancer, head and neck squamous cell cancer, ovarian cancer, colon cancer, cholangiocarcinoma and cervical cancer (4,5). Enhanced expression of this integrin is associated with poor prognosis in colon cancer and oral cancer $(4,6)$. However, the role of $\alpha v \beta 6$ integrin in breast carcinoma is still controversial.

Integrins bind to extracellular matrix (ECM) glycoproteins including collagens, fibronectins (FNs), laminin, and control biological and cellular functions such as cell adhesion, migration, invasion, proliferation, differentiation and apoptosis (4). $\alpha v \beta 6$ integrin binds $\mathrm{FN}$ in an RGD-dependent manner (7). FN has been implicated in the development of multiple types of human cancer $(8,9)$ and is associated with cell migration and invasion in metastatic models $(9,10)$. In breast cancer, FN has shown higher expression than that in normal breast parenchyma, with a different distribution (11).

Molecules of the ECM can be degraded by proteolytic enzymes, such as matrix metalloproteinases (MMPs) (12). Degradation of ECM is precisely regulated under normal physiological conditions, but when dysregulated it causes many diseases such as cancer, arthritis, and fibrosis (13). MMP-9 expression is often upregulated and the upregulation is associated with a shortened relapse-free survival in breast cancer patients (14). Previous studies have shown that FN can be degraded into fragments in vitro by MMP-9 (15); therefore, the role of degraded FN in breast cancer progression should be clarified.

Here, we examined the expression of $\beta 6$ integrin using immunohistochemistry in breast cancer samples, investigated its correlation with clinicopathological disease variables and evaluated its prognostic value. Furthermore, we assessed the influence of MMP-9-degreaded FN on the regulation of $\beta 6$ integrin and breast cancer progression by incubating MCF-7 human breast cancer cells with MMP-9-degraded FN.

\section{Materials and methods}

Ethics statement and patient samples. One hundred and twelve breast cancer samples from patients who underwent 
surgery for primary breast cancer between 2003 and 2010 at the Affiliated Hospital of Weifang Medical University were evaluated in this study. Sixteen breast tissue samples from individuals confirmed to be free of malignancy were analyzed for negative control purposes. All patients received no treatment before surgery. The human breast cancer samples were obtained following written informed consent, and the protocols were approved by the Ethics Review Committee of the World Health Organization of the Collaborating Center for Research in Human Production and authorized by the Weifang Medical University.

Digestion of $p F N$. Pro-MMP-9 was obtained from Calbiochem (VWR International, Poole, UK). Following activation, MMP-9 was incubated with $10 \mu \mathrm{g} / \mathrm{ml} \mathrm{pFN}$ (Sigma, St. Louis, MO, USA) at a ratio of 1:10. The pFN/MMP-9 mixtures were incubated at $37^{\circ} \mathrm{C}$ for $48 \mathrm{~h}$, and then $20 \mu \mathrm{l}$ was removed and added to double non-reducing sample buffer to stop the reaction. Samples were run on an SDS $10 \%$ acrylamide gel, and stained with Coomassie blue.

Cell culture. The human breast cancer MCF-7 cells were cultured in RPMI-1640, and the MDA-MB-231, MDA-MB-468, SK-BR-3, MDA-MB-453, T47D and MCF-10A cell lines were grown in Dulbecco's modified Eagle's medium (DMEM) supplemented with $10 \%$ FBS. The cells were incubated at $37^{\circ} \mathrm{C}$ in humidified air with $5 \% \mathrm{CO}_{2}$. All the cells were obtained from the Typical Culture Collection of the Chinese Academy of Science (Shanghai, China). Cell lines were authenticated on the basis of viability, and short tandem repeat (STR). The anti- $\beta 6$ integrin antibody (Santa Cruz Biotechnology, Santa Cruz, CA, USA) blocks the function of $\beta 6$ integrin at a concentration of $100 \mu \mathrm{g} / \mathrm{ml}$. For the treated cells, cells were cultured in medium containing MMP-9-degraded FN (50 ng/ml) and/or $5 \mathrm{ng} / \mathrm{ml}$ TGF- $\beta 1$ (Sigma).

Quantitative RT-PCR. Gene expression was assessed by quantitative real-time reverse transcription-PCR (qPCR). Housekeeping gene, GAPDH, was detected to ensure accurate measurement. The details of the primers for $\beta 6$ integrin and GAPDH were previously reported (16). Total RNA was extracted, and reverse transcriptase reaction was manipulated using SuperScript One-Step RT-PCR kit (Invitrogen, Carlsbad, CA, USA) according to the manufacturer's instructions. Relative mRNA expression of the treated group to the nontreated one was calculated using the comparative $\mathrm{Ct}$ method and compared with a calibrator (17).

RNA interference. MCF-7 breast cancer cells were transfected with $\beta 6$ integrin siRNA, focal adhesion kinase (FAK) siRNA or control non-specific siRNA according to the manufacturer's instructions. Cells were briefly grown in $35-\mathrm{mm}$ dishes and transfected with the siRNAs at a concentration of $200 \mathrm{pmol} / \mathrm{well}$. Depletion of $\beta 6$ integrin and FAK was determined by western blotting.

Invasion assay. Cell invasion through a three-dimensional ECM was assessed using BD Matrigel invasion chambers (BD Biosciences, Bedford, MA, USA). Transwell membranes were coated with $50 \mu \mathrm{g} / \mathrm{ml}$ human cellular FN or
MMP-9-degraded FN as previously described (18). Cells were resuspended and plated into the chamber inserts. For antibodyblockage studies, the cells were seeded in medium containing anti- $\beta 6$ integrin. After $24 \mathrm{~h}$, the cells invading the filters were fixed, stained, and counted. At least 3 separate microscopic fields were counted per membrane.

Immunohistochemistry. The standard avidin-biotin indirect immunoperoxidase method was adopted for immunohistochemistry using routinely processed paraffin-embedded specimens. The sections were incubated with the primary antibodies overnight at $4^{\circ} \mathrm{C}$, and then incubated with biotinylated secondary antibodies and the avidin-peroxidase complex. The peroxidase reaction was visualized by incubation with DAB.

Immunofluorescence. For immunofluorescence staining, a total of $2 \times 10^{4}$ cells were plated onto glass coverslips and grown for $48 \mathrm{~h}$ before fixation with $4 \%$ paraformaldehyde. Cells were permeabilized with $0.25 \%$ Triton X-100. Slides were then stained with fluorescein-conjugated secondary $\mathrm{Ab}$ for $30 \mathrm{~min}$ at $25^{\circ} \mathrm{C}$ after incubation with the primary antibody. Hoechst 33258 dye ( $1 \mu \mathrm{g} / \mathrm{ml}$; Sigma) was subsequently used to stain the nuclei.

Flow cytometry. Confluent cells (80\%) were harvested and resuspended in HEPES buffer. Cells were incubated with mouse antibody against human $\beta 6$ integrin. After $30 \mathrm{~min}$ at $4^{\circ} \mathrm{C}$, the cells were washed and stained with a R-phycoerythrin-labeled goat anti-mouse-IgG [F(ab)2-fragment; Dianova]. Flow cytometry was performed using a FACSCalibur system (Becton-Dickinson, Heidelberg, Germany).

Isolation of tumor cell variants. Tumor cell variants with high and low $\beta 6$ integrin expression were isolated from the parental MCF-7 breast cancer cells using a cell sorter. Single cells were plated into 96-well plates and expanded. The expression of $\beta 6$ integrin was measured by flow cytometry. Repeated measurements of the cell surface expression of $\beta 6$ integrin confirmed that the stability of the $\beta 6$ integrin expression phenotype of the subcell lines remained for at least 50 generations.

Immunoprecipitation analysis. For immunoprecipitation in vitro, the MMP-9-degraded FN and cell lysate of MCF-7 were mixed in binding buffer. Immunoprecipitations were carried out indirectly using anti- $\beta 3$ and $-\beta 5$ (B-10) (both from Santa Cruz Biotechnology), $\beta 6$ integrin or isotype-matched control IgG (Sigma) and captured with protein $\mathrm{G}$ agarose beads (Thermo Fisher Scientific Inc., Rockford, IL, USA). Extracts and precipitates were analyzed by western blotting.

Western blot analysis. Samples $(30 \mu \mathrm{g})$ of the cellular proteins were loaded on $12 \%$ SDS-polyacrylamide gel. Separated proteins were transferred to PVDF membranes. The membranes were incubated with primary antibodies, followed by secondary antibodies (Maxim Biotechnology, Fuzhou, China). Detection was performed using ECL chemoluminescence (ECL System; Amersham International, Little Chalfont, UK). $\beta$-actin was used as the control (diluted 1:1,000; Chemicon, Temecula, CA, USA). The membranes were scanned and the intensity of the bands were analyzed using Gel-Pro analyzer software. 
A
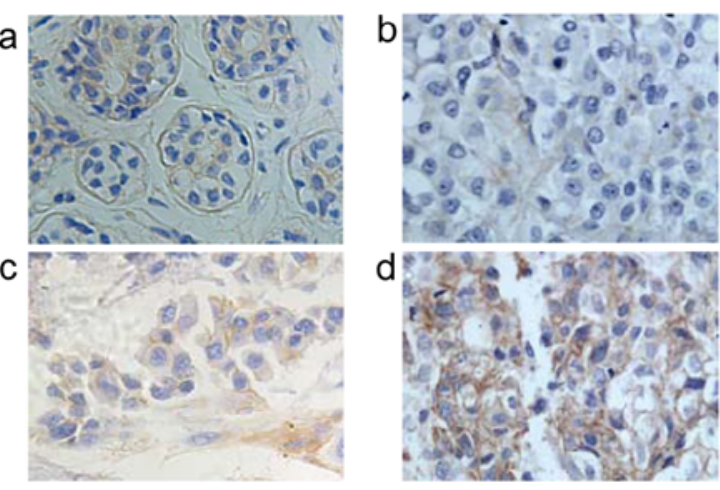

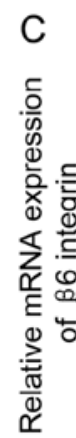
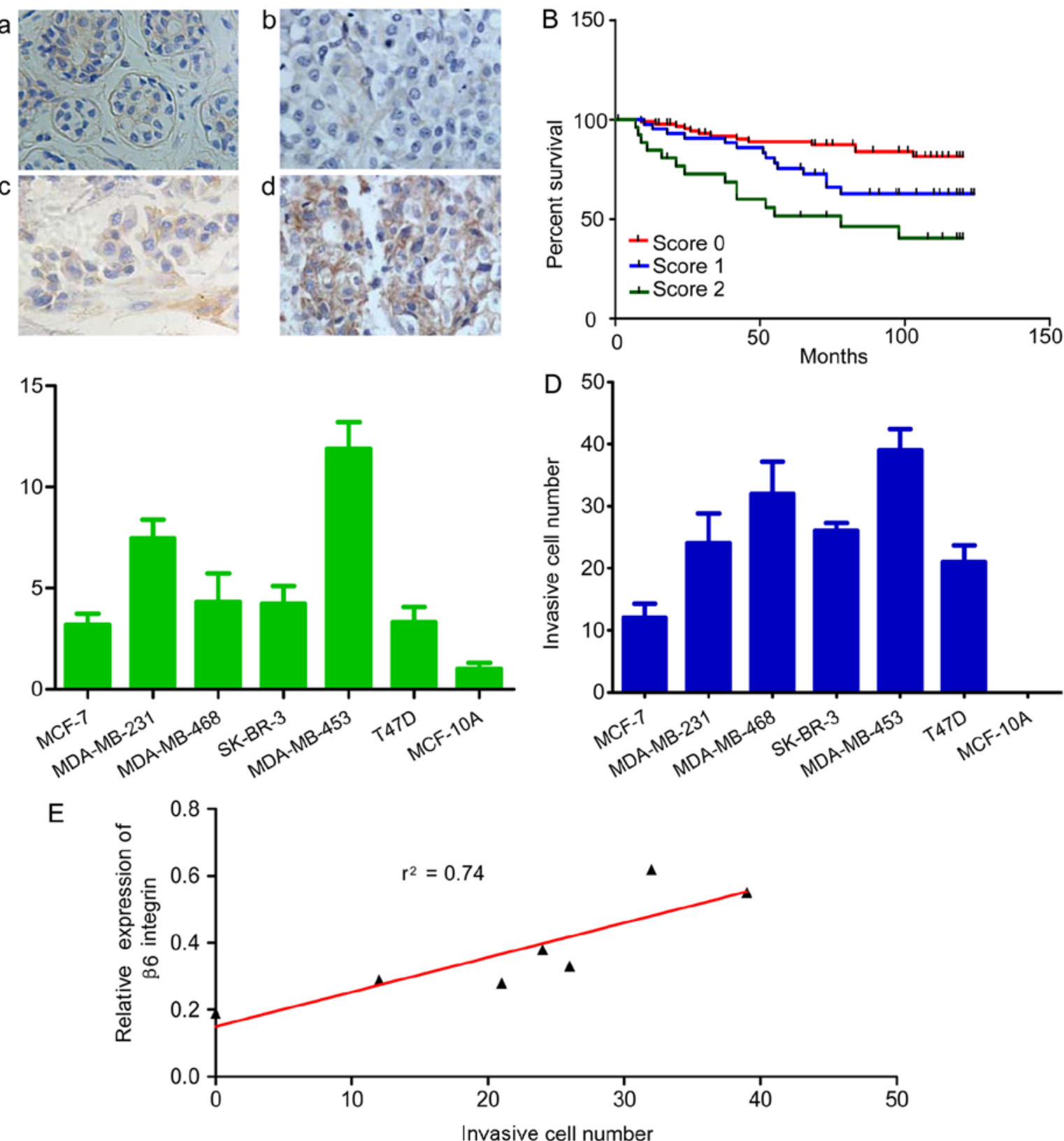

Figure 1. Predictive value of $\beta 6$ integrin for the prognosis of breast cancer patients. (Aa-d) $\beta 6$ integrin expression in normal duct and breast cancer samples. (B) Kaplan-Meier survival analysis using the log-rank test for overall survival of the breast cancer patients. (C) The mRNA expression of $\beta 6$ integrin in different breast cancer cell lines as shown by qPCR. (D) Cell invasiveness of breast cancer cell lines as determined by matrix invasion assay. (E) The linear relationship between invasive cell number and relative expression of $\beta 6$ integrin $\left(r^{2}=0.74\right)$.

Statistical analysis. SPSS v20.0 was used for statistical analysis. Student's t-test was used to determine the significance of two groups, respectively. Spearman's correlation was used to identify the correlation between the expression of $\beta 6$ integrin and invasive cell number. $\mathrm{P}<0.05$ was considered to indicate statistically significant differences.

\section{Results}

$\beta 6$ integrin is highly correlated with the prognosis of breast cancer patients. Expression of $\beta 6$ integrin in breast cancer tissues was analyzed by immunohistochemical staining (score 0 , no expression; 1, low expression; 2 , high expression) (Fig. 1Aa-d). Kaplan-Meier plot is shown in Fig. 1B.
There was prominent survival difference between groups with score 0 and score $2(\mathrm{P}=0.092)$, as well as a survival difference in patients with score 1 and score $2(\mathrm{P}=0.073)$ as determined by the log-rank test. Furthermore, the Kaplan-Meier plot indicated that patients with score 0 or 1 had a longer survival period than patients with score 2 .

Invasive cancer cells display high $\beta 6$ integrin expression. Cell invasiveness of 6 breast cancer cell lines and MCF-10A was determined by the matrix invasion assay. The invasiveness of each cell line was quantified as the invasive cell number (Fig. 1D). Expression of $\beta 6$ integrin in these cell lines was evaluated by qPCR (Fig. 1C). We presented a logistic regression-based method for identifying the relation- 


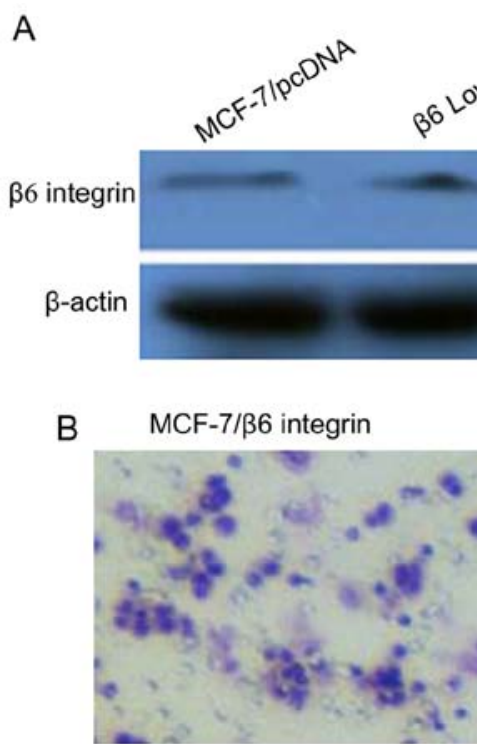

$\beta 6$ integrin low

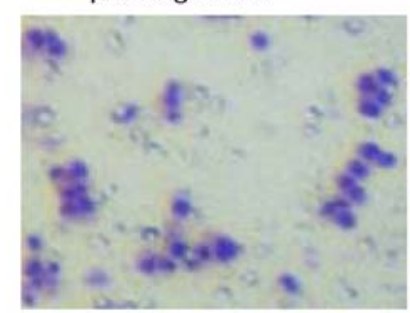

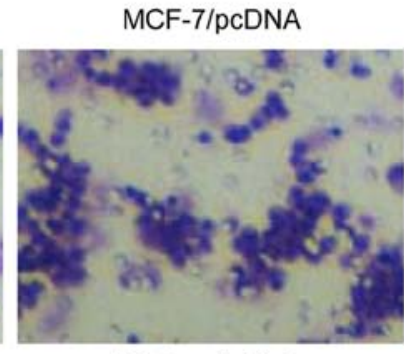

$\beta 6$ integrin high

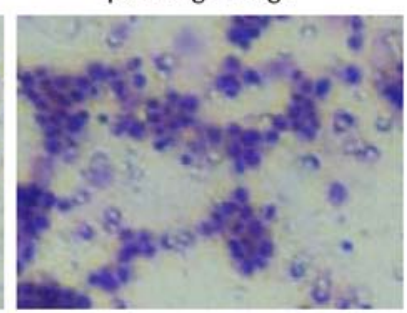

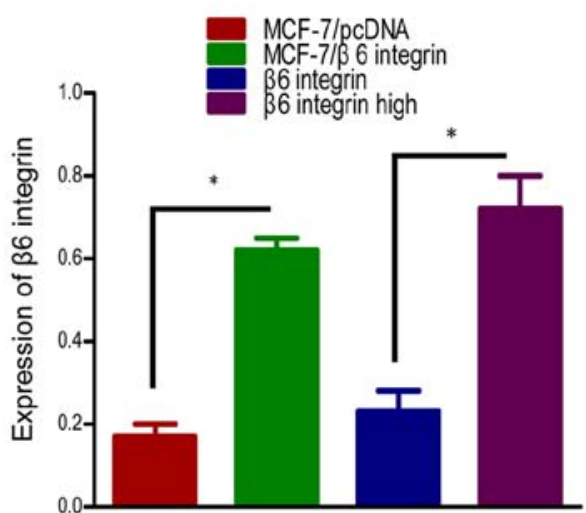

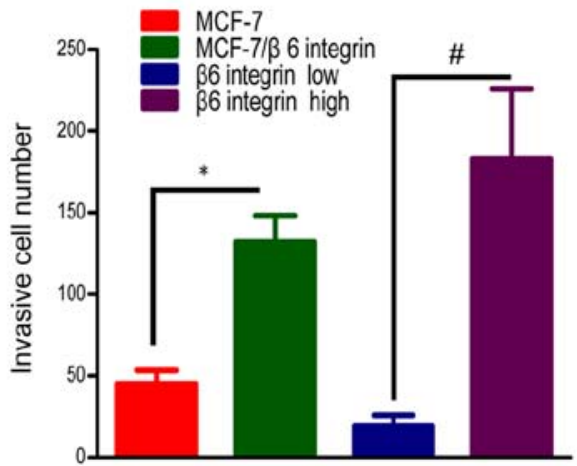

Figure 2. $\beta 6$ integrin facilitates cell invasion. (A) Higher expression of $\beta 6$ integrin in MCF-7/ $\beta 6$ integrin cells and the $\beta 6$ high subcell line. (B) MCF-7/ $\beta 6$ integrin and $\beta 6$ high cells exhibited a higher number of invasive cells. ${ }^{*} \mathrm{P}<0.05,{ }^{"} \mathrm{P}<0.01$.

ship between expression of $\beta 6$ integrin and invasive cell number and there was significant linear relationship (Fig. 1E, $\left.r^{2}=0.7413\right)$.

High $\beta 6$ integrin expression leads to enhanced cell invasion. MCF-7//36 integrin cells exhibited a much higher fluorescence intensity (data not shown). Western blotting revealed that the difference in the expression of $\beta 6$ integrin was 4.3 -fold between these two cell lines (Fig. 2A). The cell invasiveness of the MCF-7/ 36 integrin cells was higher than that of cells transfected with the pcDNA plasmids (Fig. 2B). We next isolated two subcell lines that expressed either high or low amounts of $\beta 6$ integrin from MCF-7 cells using a cell sorter. The stable $\beta 6$ integrin expression levels were confirmed during culture by cytofluorometry (data not shown). $\beta 6$ integrin expression of the isolated $\beta 6$ high subcell line was significantly increased to $>7.3$ times the value of the $\beta 6$ low group as shown by western blotting (Fig. 2A). Results of the invasion assay revealed that the invasiveness of the $\beta 6$ high group was markedly higher than that of the $\beta 6$ low group (Fig. 2B).

MMP-9-dFN treatment upregulates $\beta 6$ expression. MMP-9 was localized in the front breast cancer cells (Fig. 3A). Activated MMP-9 was incubated with plasma FN. After $48 \mathrm{~h}$, a clear band was visible at the level of $120 \mathrm{kDa}$ (Fig. 3B). A significant increase in $\beta 6$ integrin mRNA was observed in the MMP-9-dFN and the MMP-9-dFN/TGF- $\beta 1$-treated cells, whereas the native FN group also showed a similar tendency but not as conspicuous (Fig. 3C). Immunoblotting demonstrated that the protein levels of $\beta 6$ integrin subunit were also increased significantly after TGF- $\beta 1$ treatment and more prominent following treatment with the combination of MMP-9-dFN/TGF- $\beta 1$. Expression of $\beta 6$ integrin after the combined treatment with MMP-9-dFN/TGF- $\beta 1$ was significantly increased to $>2$-fold of the value of the TGF- $\beta 1$-treated group, and 4-fold of the MMP-9-dFN-treated group (Fig. 3D).

$\alpha v \beta 6$ integrin heterodimers form following disruption of heterodimers of $\alpha v \beta 3$ and $\alpha v \beta 5$ by MMP-9-dFN. Immunofluorescence of the $\beta 6$ integrin subunits in MCF-7 cells showed patchy staining of bottom surfaces of cell clusters at the cell peripheries (Fig. 3E). After FN treatment, the staining appeared in larger adhesion plaques of the spreading cells, and was intensely positive in larger adhesion plaques after treatment with MMP-9-dFN (Fig. 3E). Immunoprecipitation revealed enhanced levels of $\beta 6$ integrin heterodimers co-precipitating with the $\alpha \mathrm{v}$ subunit in the MMP-9-degraded FN-treated cells with depressed $\beta 3$ and $\beta 5$ integrin, whereas FN could not regulate the $\alpha v \beta 6$ integrin heterodimers (Fig. 3F).

Promotion of the migration and invasion of MCF-7 cells by MMP-9-dFN through FAK phosphorylation. The MCF-7 cells were significantly more chemotactic when the Transwells were coated with MMP-9-degraded FN than when they were not coated or were coated with FN (Fig. 4A). MMP-9$\mathrm{dFN}$-mediated migration was inhibited by a function blocking $\beta 6$ integrin antibody. Transient knockdown of FAK by specific siRNA in the MCF-7 cells blocked invasion induced by 

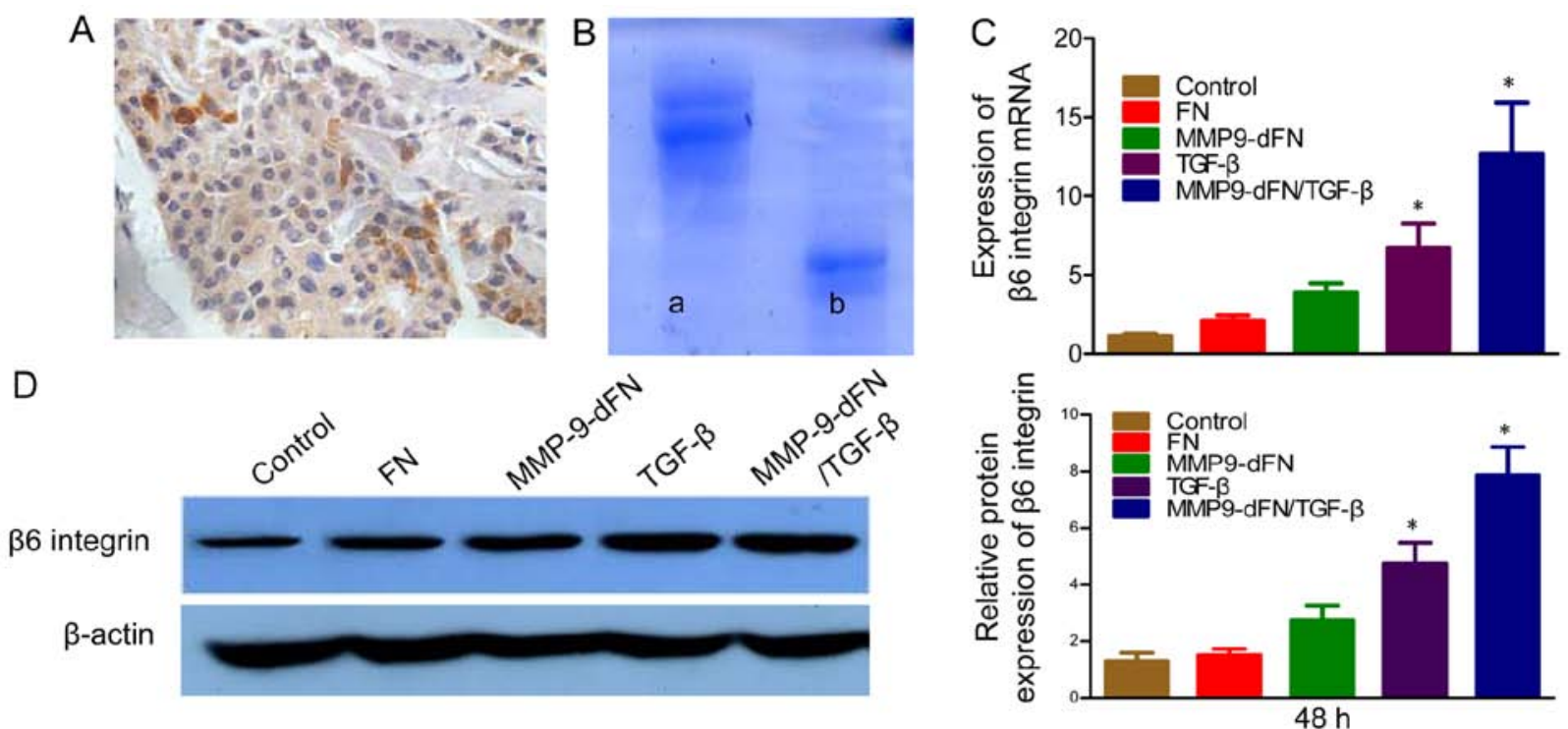

E
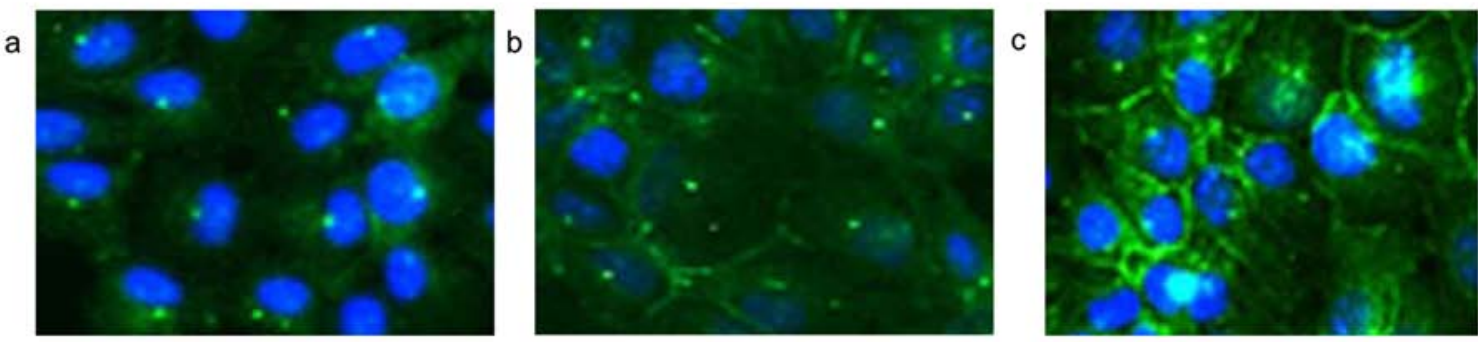

F

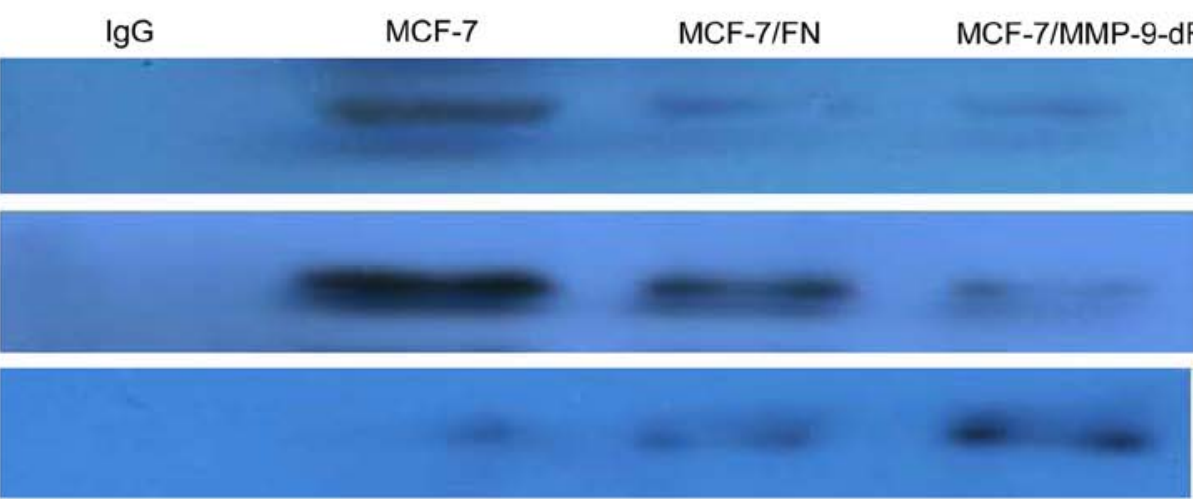

$\beta 3$ integrin

$\beta 5$ integrin

$\beta 6$ integrin

Figure 3. Influence of MMP-9-dFN on $\beta 6$ integrin expression. (A) MMP-9 localizes in the front cancer cells. Some inflammatory cells also expressed MMP-9. (B) FN was cleaved into an $\sim 120 \mathrm{kDa}$ fragment by MMP-9 (a, native FN; b, degraded-FN). (C) Immunoblotting analyses of $\beta 6$ integrin in the MCF-7 cells after treatments with MMP-9-dFN and TGF- $\beta 1$. (D) MMP-9-degraded FN significantly elevated $\beta 6$ integrin. (E) Immunofluorescence expression of $\beta 6$ integrin (a, control cells; b, FN-treated cells; c, MMP-9-dFN treated cells). (F) Immunoprecipitation shows a prominent increase in the co-precipitated $\beta 6$ integin subunit in the MMP-9-dFN cells. " $\mathrm{P}<0.05$. FN, fibronectin; MMP, matrix metalloproteinase.

MMP-9-dFN (Fig. 4A). Western blotting of the cell lysates revealed high expression levels of pFAK Ser732 and pFAK Ser722 in the MMP-9-degraded FN-treated cells (Fig. 4B). FAK was found to be phosphorylated on Thr567/577 in both the MMP-9-dFN- and FN-treated cells (Fig. 4B). Inhibition of Src by PP2 blocked the phosphorylation of FAK at its Ser732, Ser722, and Tyr576 residues (Fig. 4C) and cell invasion induced by MMP-9-dFN (Fig. 4D).

MMP-9-dFN induces MCF-7 migration and invasion through crosstalk with FAK-Src, Src-related Erk and PI3K/Akt/Smad-1/5/8 activation. MCF-7 cells treated with MMP-9-dFN displayed elevated phosphorylation of Src, Erk1/2, and PI3K/Akt in a dose-dependent manner (Fig. 5A).
As shown in Fig. 5B, FAK-associated phosphorylation of Src, Erk1/2 and PI3K/Akt was reduced in the FAK-knockdown cells. LY294002 (PI3K inhibitor) markedly lowered the phospho-Smad-1/5/8 protein modulated by MMP-9-dFN, and reduced phospho-Smad-1/5/8 staining in the nucleus. However, PD98059 (Erk pathway inhibitor) did not significantly change the expression of phospho-Smad-1/5/8 and the nuclear distribution (Fig. 5C and D).

\section{Discussion}

Breast cancer is commonly associated with marked alteration in ECM architecture and composition, especially in ECM proteins functioning as ligands $(19,20)$. Knowledge of how 
A

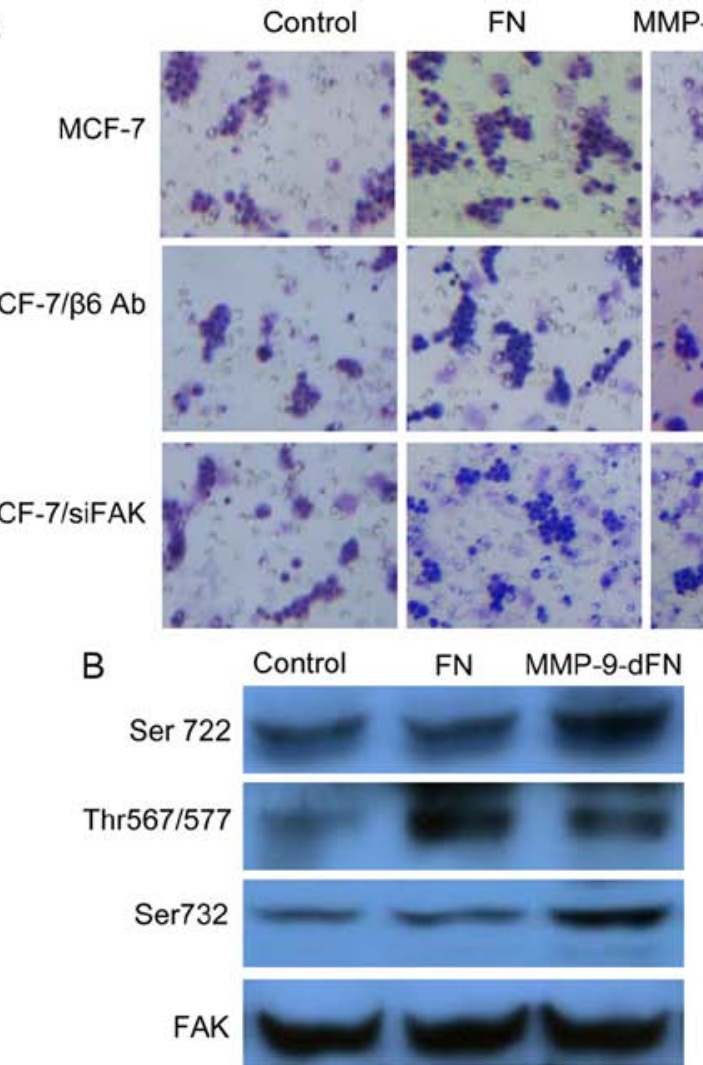
MMP-9-degraded FN

C
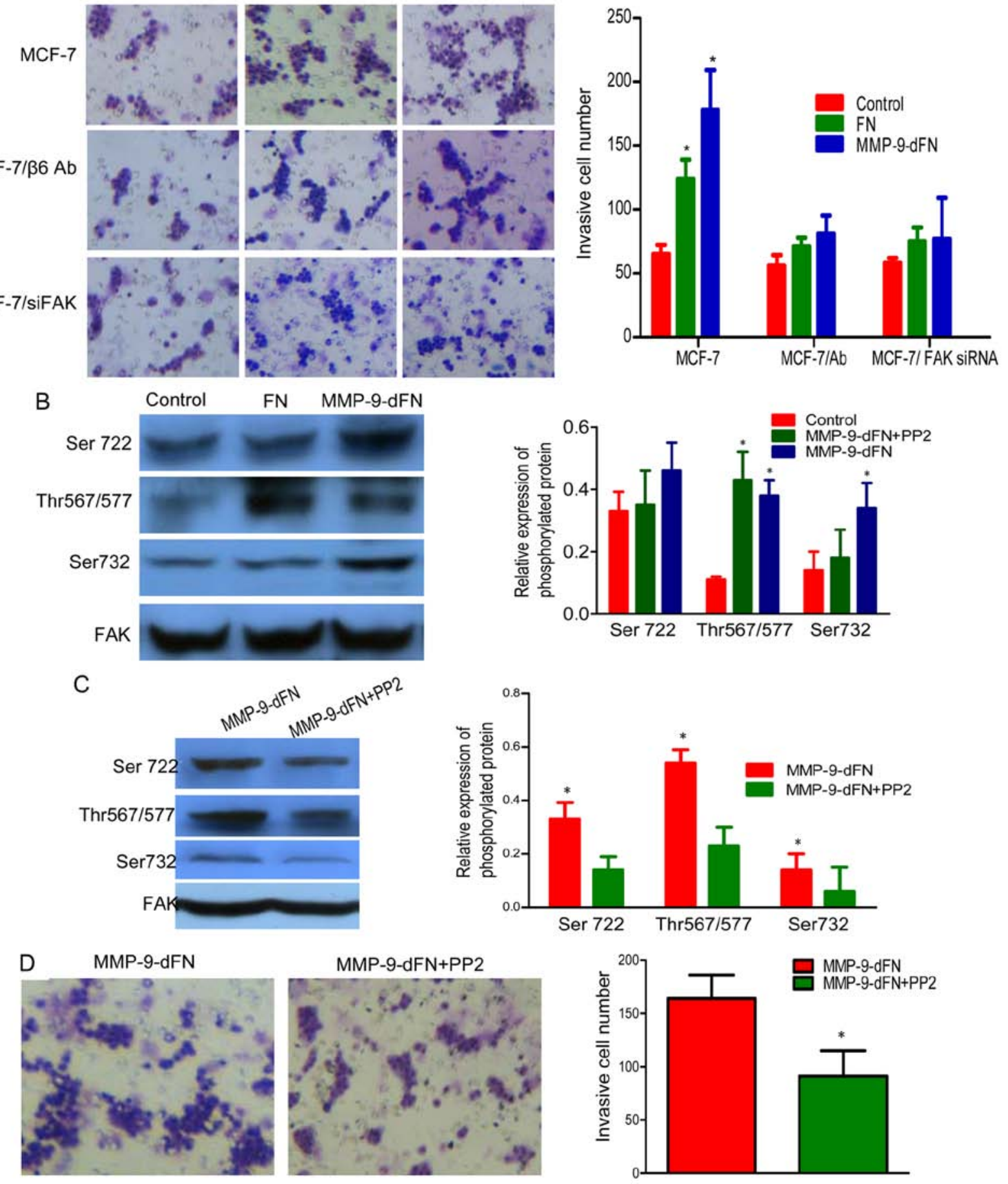

Figure 4. MMP-9-dFN promotes migration and invasion through the phosphorylation of FAK. (A) Knockdown of FAK by siRNA reduced the invasive ability of the cells. (B) p-FAK Tyr576 was increased in the FN- and MMP-9-dFN treated cells. (C) Inhibition of Src by PP2 blocked the phosphorylation of FAK at its Ser732, Ser722 and Tyr576 residues. (D) Inhibition of Src by PP2 prevented cell invasion induced by MMP-9-dFN. "P<0.05. MMP, matrix metalloproteinase; FAK, focal adhesion kinase.

ECM composition and topography are maintained and how ECM deregulation influences cancer progression may help develop new therapeutic interventions by targeting the tumor niche. Among the ECM components, FN has been recognized as a key element in promoting cell adhesion and migration of many cell types. In fact, FN has been implicated in the metastatic phenotype of multiple types of human cancers (9), and it is also associated with cell migration and invasion in several metastatic models $(10,21)$. FN in breast carcinomas has stronger expression than that in normal breast parenchyma, with a different distribution (22).

Abnormal ECM affects cancer progression by directly promoting cellular transformation and metastasis (23). Confocal scanning laser microscopy revealed that co-labeled patterns of MMP-9 with FN and the interaction between MMP-9 and FN contribute to FN degradation (24). Our experiments also showed that MMP-9 localized to the cytoplasm of the front cancer cells, suggesting that MMP-9 produced 
A

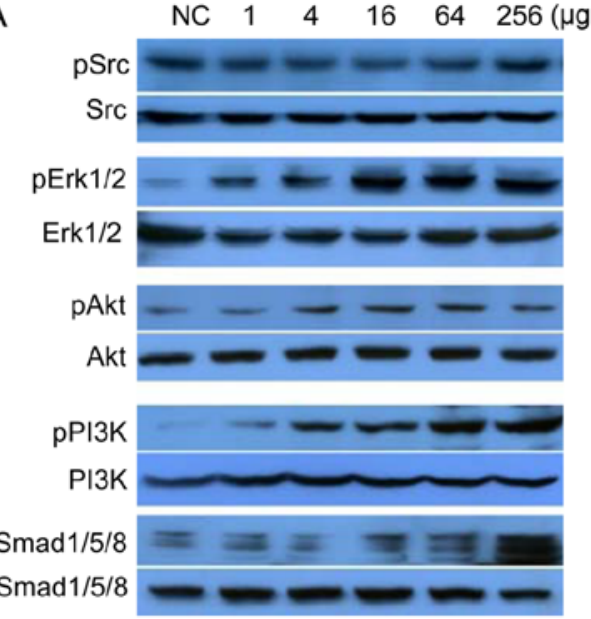

B

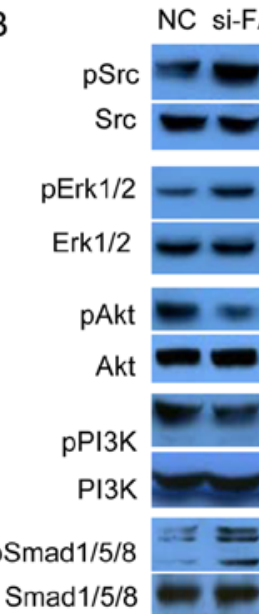

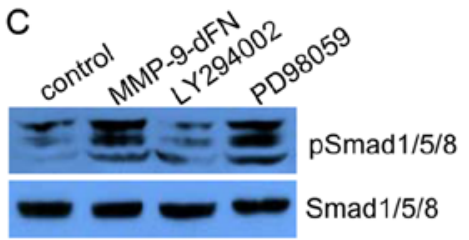

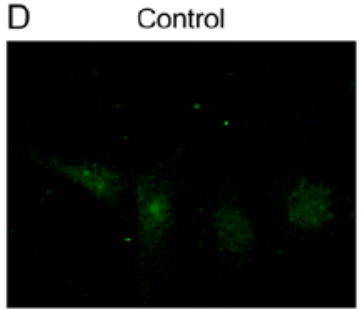

LY294002

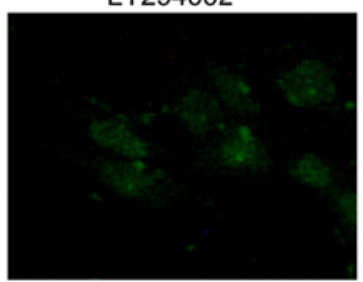

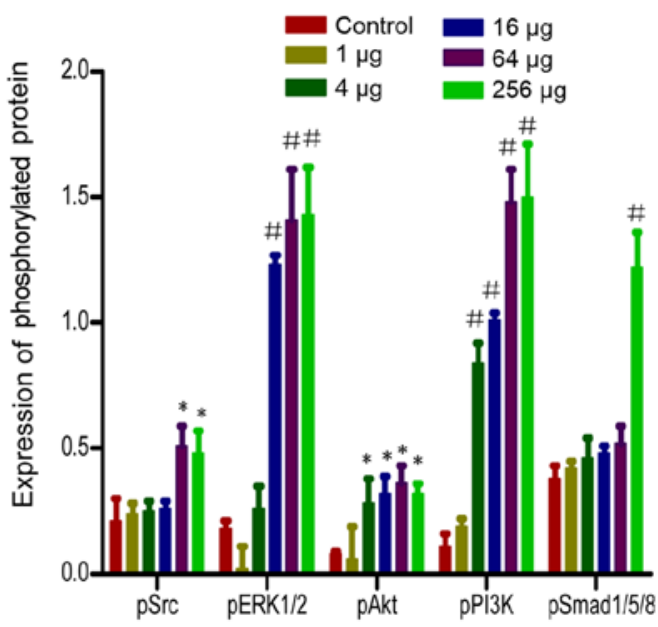

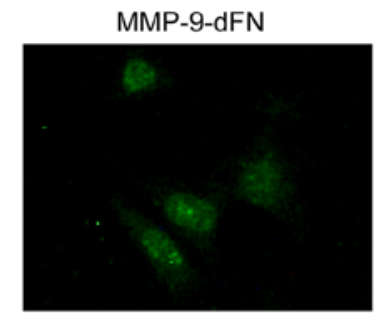

PD98059

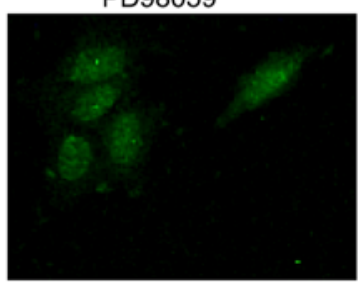

Figure 5. Migration and invasion induced by MMP-9-dFN occur through Src-related Erk and PI3K/Akt/Smad-1/5/8. (A) MMP-9-degraded FN caused increased phosphorylation of Src, Erk1/2, PI3K/Akt and Smad-1/5/8 in a dose-dependent manner in MCF-7 cells. (B) Inhibition of FAK reduced the phosphorylation of Src, Erk1/2, PI3K/Akt and Smad-1/5/8 in the MCF-7 cells. (C) LY294002 (PI3K inhibitor) blocked the phospho-Smad-1/5/8 induced by MMP-9-degraded FN in MCF-7 cells. (D) Nuclear translocation of phospho-Smad-1/5/8 induced by MMP-9-dFN was blocked by inhibition of PI3K. However, PD98059 (Erk pathway inhibitor) did not significantly change the expression of phospho-Smad-1/5/8 and the nuclear distribution. ${ }^{*} \mathrm{P}<0.05$, ${ }^{~} \mathrm{P}<0.01$. MMP, matrix metalloproteinase; FN, fibronectin; FAK, focal adhesion kinase

by cancer cells has contact with FN distributed in the stroma leading to FN degradation. Thus, it was hypothesized that MMP-9 cleaves FN and contributed to the migration and progression of breast cancer. In this study, MCF-7 cells were significantly more chemotactic when the Transwell plates were coated with MMP-9-degraded FN than when they were not coated or coated with FN.

Interactions between $\mathrm{FN}$ and integrins play an important role in tumor cell migration, invasion and metastasis (25). $\alpha v \beta 6$ integrin was found to be significantly upregulated in certain cancer types including colon $(18,26)$ and ovarian carcinoma (27) and in early stage non-small cell lung cancer (NSCLC) (28). In the present study, $\beta 6$ integrin expression was upregulated in many breast cancer samples and high expression of $\beta 6$ integrin was found to be associated with poor prognosis. Expression of $\beta 6$ integrin in several breast cancer cell lines was also determined by immunoblotting. A significant linear relationship between invasive cell number and relative expression of $\beta 6$ integrin was established.

$\alpha v \beta 6$ integrin was found to enhance the tumorigenic features of colon carcinoma, including activation of autocrine
TGF- $\beta$ and migration on interstitial FN. After MMP-9degraded FN treatment, the staining of $\beta 6$ integrin was intensely positive in larger adhesion plaques of spreading cells. Furthermore, a marked inductive formation of $\alpha v \beta 6$ integrin heterodimers occurred during treatment of MMP-9-degraded $\mathrm{FN}$ at the expense of disrupting heterodimers of $\alpha \mathrm{v} \beta 3$ and $\alpha v \beta 5$ integrin. These results indicate that MMP-9-degraded FN may adjust components of the $\alpha v$ heterodimers.

FAK, a cytoplasmic non-receptor tyrosine kinase signaling protein, has recently been established as a key component of the signal transduction pathway triggered by interaction with $\beta$ integrin. Our results showed high expression levels of pFAK Ser732, pFAK Ser722, and pFAK Tyr576 after treatment with MMP-9-degraded FN. Phosphorylation of FAK at its Ser732, Ser722, Tyr576 residues induced by MMP-9-degraded FN was obviously reduced upon treatment with the Src inhibitor. The signaling pathways downstream of FAK are complex and roughly divided into the FAK-Src-related Ras-MEKMAPK and PI3K/Akt signaling pathways. It appears that FAK promotes FN-mediated cancer metastasis through the activation of Src, Erk1/2 and PI3K/Akt. In the present study, 
FAK-associated phosphorylation of Erk1/2, and PI3K/Akt was reduced in the FAK-knockdown cells.

It has been established that activation of integrins and cell signaling through PI3K/Akt are followed by the phosphorylation of signal transducer Smad-1/5/8 and the regulation of target genes (29). The phosphorylated Smad1 and Smad5 form heterooligomeric complexes with Smad4 and then translocate into the nucleus to activate the transcription of target genes (30). After the treatment of MMP-9-degraded FN, intensive phospho-Smad-1/5/8 staining was observed. In addition, phospho-Smad-1/5/8 was markedly weakened when PI3K was inhibited. In brief, those results indicate that phospho-Smad-1/5/8 induced by MMP-9-degraded FN was PI3K/Akt-dependent.

In conclusion, we report that the protein expression profile of $\beta 6$ integrin is statistically correlated with the poor prognosis of breast cancer patients. The present study demonstrated that MMP-9-degraded FN promoted the invasion and migration of breast cancer through interaction with $\alpha v \beta 6$ involving the crosstalk between FAK-Src and Src-regulated Erk1/2 and PI3K/Akt/Smad-1/5/8 signaling. Since elevated $\beta 6$ integrin expression is predictive of outcome in early-stage breast cancer, the choice of an optimal integrin ligand is an aspect of paramount importance in the design of integrin-based targeted therapies for breast cancer.

\section{Acknowledgements}

The present study was supported by grants from the National Nature Scientific Foundation of China (nos. 30901779 and 81471048) and The Natural Science Foundation of Shandong Province (BS2011YY060).

\section{References}

1. Guo W and Giancotti FG: Integrin signalling during tumour progression. Nat Rev Mol Cell Biol 5: 816-826, 2004.

2. Mierke CT, Frey B, Fellner M, Herrmann M and Fabry B: Integrin $\alpha 5 \beta 1$ facilitates cancer cell invasion through enhanced contractile forces. J Cell Sci 124: 369-383, 2011.

3. Missan DS and DiPersio M: Integrin control of tumor invasion. Crit Rev Eukaryot Gene Expr 22: 309-324, 2012.

4. Rathinam R and Alahari SK: Important role of integrins in the cancer biology. Cancer Metastasis Rev 29: 223-237, 2010.

5. Zaidel-Bar R and Geiger B: The switchable integrin adhesome. J Cell Sci 123: 1385-1388, 2010.

6. Marelli UK, Rechenmacher F, Sobahi TR, Mas-Moruno C and Kessler H: Tumor targeting via integrin ligands. Front Oncol 3: $222,2013$.

7. van der Flier A and Sonnenberg A: Function and interactions of integrins. Cell Tissue Res 305: 285-298, 2001.

8. Zheng Y, Ritzenthaler JD, Roman J and Han S: Nicotine stimulates human lung cancer cell growth by inducing fibronectin expression. Am J Respir Cell Mol Biol 37: 681-690, 2007.

9. Waalkes S, Atschekzei F, Kramer MW, Hennenlotter J, Vetter G, Becker JU, Stenzl A, Merseburger AS, Schrader AJ, Kuczyk MA et al: Fibronectin 1 mRNA expression correlates with advanced disease in renal cancer. BMC Cancer 10: 503, 2010.

10. Jia D, Yan M, Wang X, Hao X, Liang L, Liu L, Kong H, He X, Li J and Yao M: Development of a highly metastatic model that reveals a crucial role of fibronectin in lung cancer cell migration and invasion. BMC Cancer 10: 364, 2010
11. Fernandez-Garcia B, Eiró N, Marín L, González-Reyes S, González LO, Lamelas ML and Vizoso FJ: Expression and prognostic significance of fibronectin and matrix metalloproteases in breast cancer metastasis. Histopathology 64: 512-522, 2014.

12. Fink $\mathrm{K}$ and Boratyński J: The role of metalloproteinases in modification of extracellular matrix in invasive tumor growth, metastasis and angiogenesis. Postepy Hig Med Dosw (Online) 66: 609-628, 2012 (In Polish).

13. Nagase H, Visse R and Murphy G: Structure and function of matrix metalloproteinases and TIMPs. Cardiovasc Res 69: 562-573, 2006.

14. Vizoso FJ, González LO, Corte MD, Rodríguez JC, Vázquez J, Lamelas ML, Junquera S, Merino AM and García-Muñiz JL: Study of matrix metalloproteinases and their inhibitors in breast cancer. Br J Cancer 96: 903-911, 2007.

15. Al-Hazmi N, Thomas GJ, Speight PM and Whawell SA: The $120 \mathrm{kDa}$ cell-binding fragment of fibronectin up-regulates migration of alphavbeta6-expressing cells by increasing matrix metalloproteinase-2 and -9 secretion. Eur J Oral Sci 115: 454-458, 2007.

16. Häkkinen L, Koivisto L, Gardner H, Saarialho-Kere U, Carroll JM, Lakso M, Rauvala H, Laato M, Heino J and Larjava H: Increased expression of beta6-integrin in skin leads to spontaneous development of chronic wounds. Am J Pathol 164: 229-242, 2004.

17. Shen Z, Ye Y, Kauttu T, Seppänen H, Vainionpää S, Wang S, Mustonen $\mathrm{H}$ and Puolakkainen P: The novel focal adhesion gene kindlin-2 promotes the invasion of gastric cancer cells mediated by tumor-associated macrophages. Oncol Rep 29: 791-797, 2013.

18. Bates RC, Bellovin DI, Brown C, Maynard E, Wu B, Kawakatsu H, Sheppard D, Oettgen P and Mercurio AM: Transcriptional activation of integrin beta 6 during the epithelial-mesenchymal transition defines a novel prognostic indicator of aggressive colon carcinoma. J Clin Invest 115: 339-347, 2005.

19. Gehler S, Ponik SM, Riching KM and Keely PJ: Bi-directional signaling: Extracellular matrix and integrin regulation of breast tumor progression. Crit Rev Eukaryot Gene Expr 23: 139-157, 2013.

20. Sisci D, Aquila S, Middea E, Gentile M, Maggiolini M, Mastroianni F, Montanaro D and Andò S: Fibronectin and type IV collagen activate ERalpha AF-1 by c-Src pathway: Effect on breast cancer cell motility. Oncogene 23: 8920-8930, 2004.

21. Liu W, Cheng S, Asa SL and Ezzat S: The melanoma-associated antigen A3 mediates fibronectin-controlled cancer progression and metastasis. Cancer Res 68: 8104-8112, 2008.

22. Schultz MJ, Swindall AF and Bellis SL: Regulation of the metastatic cell phenotype by sialylated glycans. Cancer Metastasis Rev 31: 501-518, 2012

23. Lahlou $\mathrm{H}$ and Muller WJ: $\beta 1$-integrin signaling and mammary tumor progression in transgenic mouse models: Implications for human breast cancer. Breast Cancer Res 13: 229, 2011.

24. Lu CY and Lai SC: Matrix metalloproteinase-2 and -9 lead to fibronectin degradation in astroglia infected with Toxoplasma gondii. Acta Trop 125: 320-329, 2013.

25. Subbaram S and Dipersio CM: Integrin $\alpha 3 \beta 1$ as a breast cancer target. Expert Opin Ther Targets 15: 1197-1210, 2011.

26. Desgrosellier JS and Cheresh DA: Integrins in cancer: Biological implications and therapeutic opportunities. Nat Rev Cancer 10: 9-22, 2010.

27. Alam H, Kundu ST, Dalal SN and Vaidya MM: Loss of keratins 8 and 18 leads to alterations in $\alpha 6 \beta 4$-integrin-mediated signalling and decreased neoplastic progression in an oral-tumour-derived cell line. J Cell Sci 124: 2096-2106, 2011.

28. Cantor D, Slapetova I, Kan A, McQuade LR and Baker MS: Overexpression of $\alpha v \beta 6$ integrin alters the colorectal cancer cell proteome in favor of elevated proliferation and a switching in cellular adhesion that increases invasion. J Proteome Res 12: 2477-2490, 2013

29. Massagué $J$ and Xi Q: TGF- $\beta$ control of stem cell differentiation genes. FEBS Lett 586: 1953-1958, 2012.

30. Karathanasi V, Tosios KI, Nikitakis NG, Piperi E, Koutlas I, Trimis G and Sklavounou A: TGF- $\beta 1$, Smad-2/-3, Smad-1/-5/-8, and Smad-4 signaling factors are expressed in ameloblastomas, adenomatoid odontogenic tumors, and calcifying cystic odontogenic tumors: an immunohistochemical study. J Oral Pathol Med 42: 415-423, 2013. 